PROCEEDINGS OF THE

AMERICAN MATHEMATICAL SOCIETY

Volume 127, Number 5, Pages 1397-1398

S 0002-9939(99)04687-0

Article electronically published on January 28, 1999

\title{
AN ELEMENTARY PROOF OF THE PRINCIPLE OF LOCAL REFLEXIVITY
}

\author{
ANTONIO MARTÍNEZ-ABEJÓN
}

(Communicated by Palle E. T. Jorgensen)

Abstract. We give an elementary proof of the principle of local reflexivity.

We use only elementary functional analysis to give a simple and short proof of the version of the "principle of local reflexivity" proved in [2], which is an improvement of the original version given in [3]. Other short proofs can be found in [1] and [4]. We use standard notation for Banach spaces. By $X, X^{*}$ and $X^{* *}$, we denote a real or complex Banach space, its first dual and its second dual respectively; we identify $X$ with the canonical copy of $X$ contained in $X^{* *}$; given a Banach space $Y$, we write $B_{Y}:=\{y:\|y\| \leq 1\}$ and $S_{Y}:=\{y:\|y\|=1\}$; given a subset $A$ of $X, \bar{A}^{\sigma\left(X^{* *}, X^{*}\right)}$ stands for the weak $k^{*}$-closure of $A$ in $X^{* *}$ and int $A$ is the norm interior of $A$; an operator is a continuous linear function; given $\varepsilon>0$, an $\varepsilon$-isometry $T: E \longrightarrow Y$ is an operator for which $1-\varepsilon \leq\|T x\| \leq 1+\varepsilon$ for all $x \in S_{E}$.

We only require the following Lemma 1 . We omit its proof, which is an easy exercise based on the separation Hahn-Banach theorem.

Lemma 1. Let $T: X \longrightarrow Y$ be an operator, $z \in \operatorname{int} B_{X^{* *}}$ and $y \in Y$ such that $\left\|T^{* *} z-y\right\|<\varepsilon$. Then we have that $z \in \bar{L}^{\sigma\left(X^{* *}, X^{*}\right)}$, where $L:=\left\{x \in B_{X}\right.$ : $\|T x-y\|<\varepsilon\}$.

Theorem 2 (Principle of local reflexivity). Let $E \subset X^{* *}$ and $F \subset X^{*}$ be finite dimensional subspaces. Given $\varepsilon>0$ there exists an $\varepsilon$-isometry $T: E \longrightarrow X$ such that $\left.T\right|_{E \cap X}=\left.i d\right|_{E \cap X}$, and $f(T e)=e(f)$ for all $f \in F$ and all $e \in E$.

Proof. Let $\operatorname{dim} E=n$ and $\operatorname{dim} E \cap X=n-k$. Let $\left(y_{j}, h_{j}\right)_{j=1}^{n}$ be a biorthogonal system in $E \times E^{*}$ such that $\left\|y_{j}\right\|=1-\varepsilon$ and $\operatorname{span}\left\{y_{j}\right\}_{j=k+1}^{n}=E \cap X$. The identity $i d: E \longrightarrow X^{* *}$ can be given as $i d(e)=\sum_{j=1}^{n} h_{j}(e) y_{j}$. We shall find $v_{1}, \ldots, v_{k}$ in $X$ so that the operator $T: E \longrightarrow X$ defined by $T(e):=\sum_{j=1}^{k} h_{j}(e) v_{j}+$ $\sum_{j=k+1}^{n} h_{j}(e) y_{j}$ is an $\varepsilon$-isometry. Hence, the condition $\left.T\right|_{E \cap X}=\left.i d\right|_{E \cap X}$ will be satisfied automatically.

Let $W:=X^{k}$ endowed with the norm $\left\|\left(x_{j}\right)_{j=1}^{k}\right\|=\sup _{j}\left\|x_{j}\right\|$, and select $0<$ $\alpha<\min \left\{2 / 5,(1-\varepsilon)^{-1}-1, \varepsilon\left(\sum_{j=1}^{n}\left\|h_{j}\right\|\right)^{-1}\right\}$. Fix $\left\{f_{j}\right\}_{j=1}^{M}$ a basis in $F,\left\{e_{j}\right\}_{j=1}^{N}$

Received by the editors September 27, 1996 and, in revised form, August 18, 1997.

1991 Mathematics Subject Classification. Primary 46B20, 46B10.

Key words and phrases. Local reflexivity, weak*-topology, $\varepsilon$-isometry.

The author's research was supported by a postdoctoral Grant of the Ministry of Spain for Education and Science and DGYCIT Grant PB 94-1052 (Spain).

(C)1999 American Mathematical Society 
an $\alpha / 4$-net in $\operatorname{int} B_{E}$, and $\left\{u_{j}\right\}_{j=1}^{N}$ in $B_{X^{*}}$ so that $\|e\| \leq(1+\alpha) \sup _{1 \leq j \leq N}\left|e\left(u_{j}\right)\right|$ for all $e \in E$. We have that

$$
e_{j}=\sum_{r=1}^{n} \lambda_{r}^{j} y_{r}, j=1, \ldots, N .
$$

Let us write $P:=\max _{1 \leq j \leq N} \sum_{r=1}^{k}\left|\lambda_{r}^{j}\right|$ and define the set

$$
C:=\left\{\left(x_{s}\right)_{s=1}^{k} \in B_{W}:\left\|\sum_{s=1}^{k} \lambda_{s}^{j} x_{s}+\sum_{s=k+1}^{n} \lambda_{s}^{j} y_{s}\right\|<1, j=1, \ldots, N\right\} .
$$

By the above lemma, we have that $\left(y_{j}\right)_{j=1}^{k} \in \bar{C}^{\sigma\left(W^{* *}, W^{*}\right)}$. Now we set the operator $S: W \longrightarrow \mathbf{R}^{M \cdot k+N \cdot k}$ (or into $\mathbf{C}^{M \cdot k+N \cdot k}$ ) given by $S\left(\left(x_{s}\right)_{s=1}^{k}\right):=\left(f_{i}\left(x_{r}\right), u_{j}\left(x_{s}\right)\right)$ for $1 \leq i \leq M, 1 \leq r \leq k, 1 \leq j \leq N, 1 \leq s \leq k$.

Thus $S^{* *}\left(\left(y_{j}\right)_{j=1}^{k}\right) \in \overline{S(C)}$. Now, since $\bar{W}^{\sigma\left(W^{* *}, W^{*}\right)}=W^{* *}$ and $R(S)$ is closed, we have that $R(S)=R\left(S^{* *}\right)$, and then, for $0<\beta<\min \left\{1, \varepsilon(2 P)^{-1}\right\}$, we can find $\left(c_{j}\right)_{j=1}^{k} \in C$ and $\left(b_{j}\right)_{j=1}^{k} \in \beta B_{W}$ so that

$$
S^{* *}\left(\left(y_{j}\right)_{j=1}^{k}\right)=S\left(\left(c_{j}\right)_{j=1}^{k}\right)+S\left(\left(b_{j}\right)_{j=1}^{k}\right) .
$$

We take $v_{j}:=c_{j}+b_{j}$ for $j=1, \ldots, k$ in the definition of $T$. Thus, we already have the condition $f(T e)=e(f)$ for all $f \in F$ and all $e \in E$. Now, since $\left\|T e_{j}\right\| \leq$ $1+\left\|\sum_{r=1}^{k} \lambda_{r}^{j} b_{r}\right\| \leq 1+\beta P$ for $j=1, \ldots, N$, it is completely straightforward to check that $T$ is an $\varepsilon$-isometry.

\section{ACKNOWLEDGMENTS}

The author thanks the Department of Mathematics of the University of Texas at Austin for the hospitality during his visit.

\section{REFERENCES}

1. D.W. Dean, The equation $L\left(E, X^{* *}\right)=L(E, X)^{* *}$ and the principle of local reflexivity. Proc. of Amer. Math. Soc. 40 (1973), 146-148. MR 48:2735

2. W.B. Johnson, H. Rosenthal and M. Zippin, On bases, finite dimensional decompositions and weaker structures in Banach spaces. Israel J. Math. 9 (1971), 488-506. MR 43:6702

3. J. Lindenstrauss and H. Rosenthal, The $\mathcal{L}_{p}$ spaces. Israel J. Math. 7 (1969), 325-349.

4. C. Stegall, A proof of the principle of local reflexivity. Proc. of Amer. Math. Soc. 78 (1980) 154-156. MR 81e:46012

Department of Mathematics, The University of Texas at Austin, Austin, Texas 78712 Current address: Facultad de Ciencias, c/ Calvo Sotelo s.n., Universidad de Oviedo, Spain E-mail address: ama@pinon.cu.uniovi.es 